\title{
Gate-Tunable Field-Compatible Fluxonium
}

\author{
Marta Pita-Vidalø, ${ }^{1,2, *}$ Arno Bargerbos, ${ }^{1,2}$ Chung-Kai Yang, ${ }^{3}$ David J. van Woerkom, ${ }^{3}$ \\ Wolfgang Pfaff®, ${ }^{4}$ Nadia Haider $\odot,{ }^{1,5}$ Peter Krogstrup,${ }^{6}$ Leo P. Kouwenhoven, ${ }^{1,3}$ Gijs de Lange, ${ }^{3}$ and \\ Angela $\mathrm{Kou}^{4}$ \\ ${ }^{1}$ QuTech, Delft University of Technology, Delft 2628 CJ, Netherlands \\ ${ }^{2}$ Kavli Institute for Nanoscience, Delft University of Technology, Delft 2628 CJ, Netherlands \\ ${ }^{3}$ Microsoft Quantum Lab Delft, Delft 2628 CJ, Netherlands \\ ${ }^{4}$ Department of Physics and Frederick Seitz Materials Research Laboratory, University of Illinois \\ Urbana-Champaign, Urbana, IL 61801, USA \\ ${ }^{5}$ Netherlands Organisation for Applied Scientific Research (TNO), Delft 2628 CK, Netherlands \\ ${ }^{6}$ Center for Quantum Devices, Niels Bohr Institute, University of Copenhagen, Copenhagen 2100, Denmark \\ Denmark and Microsoft Quantum Materials Lab Copenhagen, Lyngby 2800, Denmark
}

(Received 22 November 2019; revised 2 October 2020; accepted 13 November 2020; published 14 December 2020)

\begin{abstract}
Hybrid superconducting circuits, which integrate nonsuperconducting elements into a circuit quantum electrodynamics (cQED) architecture, expand the possible applications of cQED. Building hybrid circuits that work in large magnetic fields presents even further possibilities, such as the probing of spin-polarized Andreev bound states and the investigation of topological superconductivity. Here we present a magneticfield compatible hybrid fluxonium with an electrostatically tuned semiconducting nanowire as its nonlinear element. We operate the fluxonium in magnetic fields up to $1 \mathrm{~T}$ and use it to observe the $\varphi_{0}$-Josephson effect. This combination of gate tunability and field compatibility opens avenues for the control of spinpolarized phenomena using superconducting circuits and enables the use of the fluxonium as a readout device for topological qubits.
\end{abstract}

DOI: 10.1103/PhysRevApplied.14.064038

\section{INTRODUCTION}

Circuit quantum electrodynamics (cQED), where photons are coherently coupled to artificial atoms built with superconducting circuits, has enabled the investigation and control of macroscopic quantum-mechanical phenomena in superconductors [1-3]. Recently, hybrid circuits incorporating semiconducting nanowires [4-7] and other electrostatically gateable elements [8-11] into superconducting circuits have broadened the scope of cQED to probing mesoscopic superconductivity $[4,5,7$, 12]. Further extending the capabilities of hybrid circuits to work in magnetic fields presents the intriguing possibility of insights into topological superconductivity [13-20].

Topological superconductivity, which has garnered much theoretical and experimental interest lately [21-24],

\footnotetext{
*m.pitavidal@tudelft.nl
}

Published by the American Physical Society under the terms of the Creative Commons Attribution 4.0 International license. Further distribution of this work must maintain attribution to the author(s) and the published article's title, journal citation, and DOI. results from the interplay between magnetism and superconductivity. In superconductor-proximitized semiconductors exposed to a large magnetic field, emergent quasiparticle states known as Majorana zero modes (MZMs) can form. Majorana zero modes are predicted to be robust to local perturbations and could thus serve as long-lived qubits. Several groups have proposed using superconducting circuits to both probe and control MZMs [14,17,18,20,25]. All of the abovementioned proposals, however, require the operation of a flux-based superconducting circuit in large magnetic fields.

Additionally, the behavior of superconductor-proximitized semiconductors exposed to a large magnetic field in the trivial phase remains an active field of research. Most experiments investigating the behavior of Andreev bound states (ABSs) in a magnetic field have been focused on the magnetic-field dependence of the switching current [26-28] with the exceptions of Refs. [29] and [30], in which ABS spectroscopy was performed up to fields of 300 and $11 \mathrm{mT}$, respectively. Energies below $20 \mu \mathrm{eV}$, which are interesting for high-transparency ABSs and MZMs, were, however, not accessible. A fluxonium that works in high magnetic fields and with semiconducting weak 
links would thus be an extremely useful tool both for investigating the magnetic field behavior of ABSs and also for coupling to topological superconductors. First, the fluxonium ground-to-excited state transitions are typically accessible at microwave frequencies over the entire flux range from 0 to $\pi$. One therefore gains access to the full energy-phase relations of the junction. Second, the Josephson energy of the junction can be known extremely precisely (to less than $0.4 \mu \mathrm{eV}$ ) and over multiple decades, which allows for the mapping of the characteristic energy of the semiconducting weak link. Finally, the fluxonium is also sensitive to quasiparticle poisoning events in individual ABSs in the junction since poisoning of individual ABSs would lead to additional spectral lines. Knowledge of these rates would greatly aid the design of Andreevbased qubits [12,31].

Moreover, the unique parameter regime of the fluxonium makes it particularly suited to detecting and controlling MZMs. A switch in the parity of MZMs coupled to the fluxonium circuit corresponds to a switch in the direction of the persistent current flowing in the fluxonium circuit. Parity switches of the MZMs would thus result in the observation of two copies of the fluxonium spectrum [25]. In addition, the presence of MZMs coupled to the fluxonium changes the periodicity of its spectrum from $2 \pi$ to $4 \pi$. In these proposals, the linewidth of the fluxonium transition determines the sensitivity with which the fluxonium could determine the presence of MZMs. Beyond its detection capabilities, multiple fluxonium devices coupled to MZMs could also implement braiding operations on the MZMs [20].

Here we have realized a hybrid fluxonium incorporating an Al-proximited nanowire that operates up to $1 \mathrm{~T}$. We build upon recent work proximitizing semiconducting nanowires to incorporate a magnetic-field compatible, electrostatically tunable weak-link junction into the fluxonium [32]. The presented fluxonium device also has a gradiometric design and is composed of $(\mathrm{Nb}, \mathrm{Ti}) \mathrm{N}$ for further field compatibility. We demonstrate in-situ gate tunability of the fluxonium Josephson energy over more than a decade. We then operate the fluxonium in fields up to $1 \mathrm{~T}$ and map out the dependence of the fluxonium Josephson energy as a function of the field. In all regimes of magnetic field and gate voltage, we observe excellent agreement between the data and a theoretical model based on a simple Hamiltonian with a few degrees of freedom. Finally, we also demonstrate the utility of the fluxonium as a probe of mesoscopic superconductivity in magnetic fields by using the measured energy-phase relation of the junction to observe the $\varphi_{0}$-Josephson effect. The ability to observe the fluxonium spectrum over a wide range of gate voltages and magnetic fields establishes the hybrid fluxonium as a superconducting circuit for exploring superconducting phenomena in a magnetic field and as a realistic readout platform for MZMs.

\section{MAGNETIC-FIELD-COMPATIBLE MATERIALS AND DESIGN}

Building a fluxonium compatible with the application of a magnetic field presents multiple challenges. The first challenge is reaching the fluxonium regime using magnetic-field-compatible materials. The fluxonium consists of a Josephson junction with Josephson energy $E_{J}$ in parallel with a linear superinductor [33], with inductive energy $E_{L}$, and a capacitor characterized by the energy $E_{C}$, as shown in Fig. 1(a). The fluxonium regime $\left(E_{L}<E_{C}<E_{J}\right)$ is achieved by shunting the junction with a large inductance. This parameter regime results in the eigenstates of the fluxonium being composed of superpositions of persistent currents in multiple directions [Fig. 1(b)]. Additionally, the chosen fluxonium parameters result in a spectrum that is observable over the entire range of applied external flux. Superconductor-insulatorsuperconductor $(S-I-S)$ Josephson junction arrays, commonly used to implement the fluxonium superinductance [33-35], cannot be used since they are incompatible with large magnetic fields. Recent work on magnetic-fieldcompatible materials with a large kinetic inductance, such as granular aluminium [36-39] and ( $\mathrm{Nb}, \mathrm{Ti}) \mathrm{N}$ [40-42], has presented a path to meeting the stringent requirements of the fluxonium superinductance. The magnetic-fieldcompatible fluxonium device is shown in Figs. 1(c)-1(f). All circuit elements except for the junction are fabricated using $(\mathrm{Nb}, \mathrm{Ti}) \mathrm{N}$, which has been demonstrated to have critical fields exceeding $9 \mathrm{~T}$ and inductances exceeding $75 \mathrm{pH} / \square$ [41]. Here we define the fluxonium by etching a 9-nm-thick sputtered $(\mathrm{Nb}, \mathrm{Ti}) \mathrm{N}$ film, which has a kinetic inductance of $41 \mathrm{pH} / \square$. The superinductance of the fluxonium is made with a 50 -nm-wide $(\mathrm{Nb}, \mathrm{Ti}) \mathrm{N}$ meander [Fig. 1(e)] in order to maximize the inductance of the device while minimizing spurious capacitances to ground. This design realizes an inductance of approximately $100 \mathrm{nH}$. The small width of the meanders additionally suppresses the emergence of lossy vortices due to out-of-plane fields, $B_{x}$, up to tens of millitesla [43]. We further mitigate the effects of these vortices by introducing vortex-pinning holes [inset, Fig. 1(c)] into the capacitor of the fluxonium readout resonator and the ground plane.

In addition to being composed of magnetic-fieldcompatible materials, for use as a detector, the fluxonium must also maintain its narrow linewidth during the application of a magnetic field. The application of a magnetic field precludes the possibility of using the magnetic shielding necessary for limiting flux noise in flux-based superconducting circuits. We address this challenge by building a gradiometric superinductance as shown in Fig. 1(d). Equal fluxes through each of the two loops generate equal currents that are canceled at the junction, rendering the fluxonium insensitive to flux noise due to sources larger than the fluxonium device. 
(a)
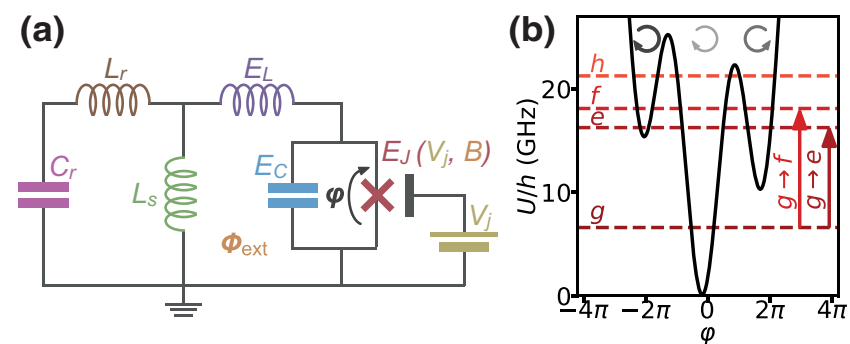

(c)

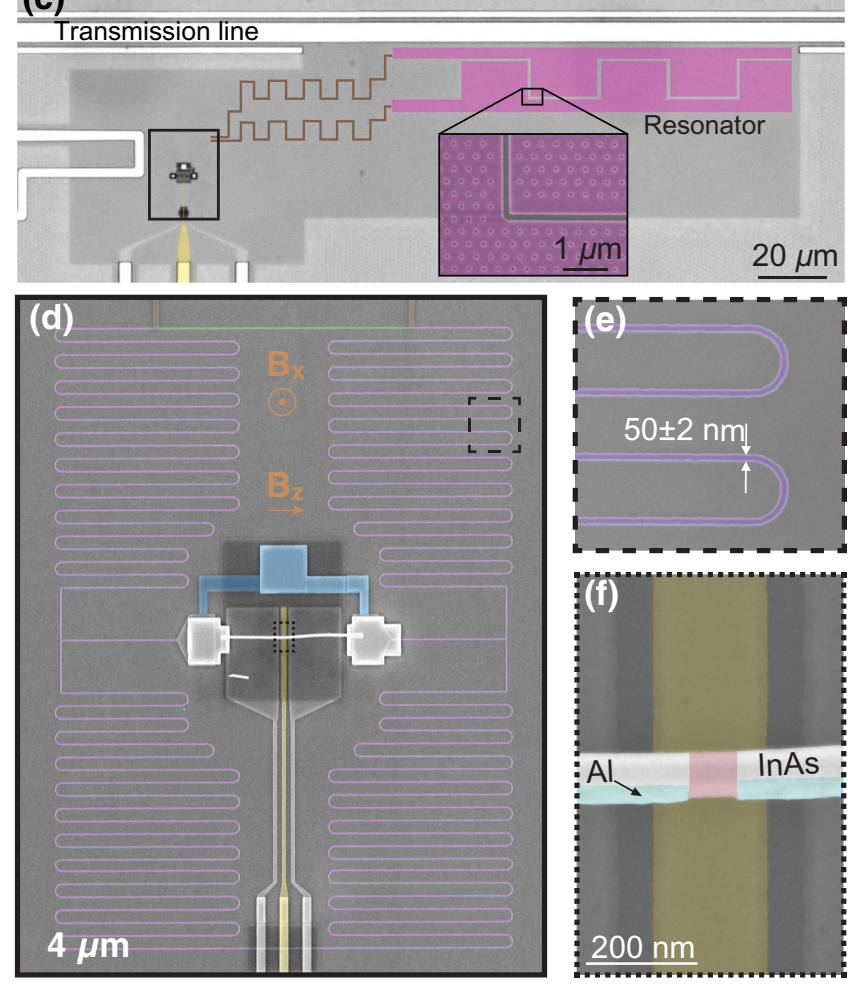

FIG. 1. Nanowire fluxonium. (a) Circuit model. The fluxonium is composed of a Josephson junction shunted by an inductor and a capacitor, which are characterized by the energies $E_{J}, E_{L}$, and $E_{C}$, respectively. The value of $E_{J}$ depends on the external magnetic field $B_{z}$ and the gate voltage $V_{j}$. A readout resonator (constituted by $L_{r}$ and $C_{r}$ ) is coupled to the fluxonium via a shared inductance $L_{s}$. (b) Potential of the fluxonium (black) versus the phase difference across the junction, $\varphi$, at $\varphi_{\mathrm{ext}}=0.2 \pi$. The lowest eigenenergies are indicated with dashed horizontal lines. Red arrows indicate transitions starting from the ground state. Circular gray arrows represent the amplitude and direction of the persistent-current states associated with different potential wells. (c) False-colored optical image showing the transmission line and the resonator, with capacitive and inductive elements shaded in pink and brown, respectively. Inset: Scanning electron microscope (SEM) image of the resonator's capacitive plates. (d) SEM image of a lithographically similar fluxonium to device $A$, corresponding to the area indicated by the box in (c). The $(\mathrm{Nb}, \mathrm{Ti}) \mathrm{N}$ superinductor [purple, enlarged in (e)], the shared inductance section (green), the parallel plate capacitor (blue), and the nanowire junction [red, enlarged in (f)] respectively correspond to the $E_{L}, L_{s}, E_{C}$, and $E_{J}$ elements in (a). The out-of-plane component of $B, B_{x}$, tunes the external magnetic flux $\Phi_{\text {ext }}$. Here $B_{z}$ is the component parallel to the wire.
Finally, a $S-I-S$ Josephson junction made of $\mathrm{Al}$ and $\mathrm{AlO}_{x}$, which has been used in all previously reported fluxonium devices, cannot be used as the $E_{J}$ element here due to its incompatibility with the magnetic field. We build a magnetic-field-compatible junction by incorporating a semiconducting InAs nanowire proximitized by an epitaxially grown 6-nm-thick aluminum layer [Fig. 1(f)] [32] into the fluxonium. The small thickness of the aluminum shell makes it resilient to magnetic fields along the wire of more than $1 \mathrm{~T}$ [32]. Similar resilience to magnetic fields could also be attained using weak links of disordered superconducting materials, such as indium oxide [44] or granular aluminum [45], as the Josephson junction. The nanowire is deterministically deposited on top of the prepatterned leads of the inductor and capacitor using a micromanipulator. The Josephson junction is then defined by etching away an Al section of approximately $80 \mathrm{~nm}$ on top of the junction gate. This small junction, however, does not provide a large enough capacitance to achieve the fluxonium regime for typical $E_{J}$ values in nanowire junctions. We thus add a parallel plate capacitor [blue in Fig. 1(d)] to decrease $E_{C}$ and achieve its required value for the fluxonium. The fluxonium capacitor consists of two square $(\mathrm{Nb}, \mathrm{Ti}) \mathrm{N}$ plates sandwiching a $29-\mathrm{nm}$-thick $\mathrm{SiN}$ dielectric. We note that this fluxonium design is flexible enough to incorporate any semiconducting material as its small junction.

\section{FLUXONIUM SPECTROSCOPY}

We first demonstrate that our device behaves as expected for a fluxonium coupled to a readout resonator. Data from two similar fluxonium devices (device $A$ and device $B$ ) will be presented in this article. We first focus on the behavior of device $A$. We monitored the transmission amplitude, $\left|S_{21}\right|$, at frequencies $f_{r \text {,drive }}$ around the resonator frequency $f_{g 0 \rightarrow g 1}$, as a function of the external phase $\varphi_{\text {ext }}=(2 e / \hbar) \Phi_{\text {ext }}$, as shown in the top panel of Fig. 2(a). Transitions are labeled as $m_{i} n_{i} \rightarrow m_{e} n_{e}$, where $m_{i}\left(m_{e}\right)$ and $n_{i}\left(n_{e}\right)$ are the initial (end) states of the fluxonium and resonator, respectively. The resonator spectrum is periodic in flux and also exhibits gaps in its visibility, which indicate that the resonator is coupled to the fluxonium. The bottom panel in Fig. 2(a) shows the flux dependence of the observed transition frequencies of the fluxonium-resonator system, measured by monitoring the transmission amplitude at $f_{r \text {,drive }}=f_{g 0 \rightarrow g 1}$ while the system is driven with a second tone with frequency $f_{f}$, drive, also via the resonator. Though the gradiometric loops that comprise the fluxonium are designed to be symmetric, the placement of the capacitor and gate lines inside them, together with inevitable imprecision when manually depositing the nanowire, lead to a small imbalance between the effective area of the two gradiometer loops. The imbalance allows us to thread a flux using an out-of-plane magnetic field, 
(a)

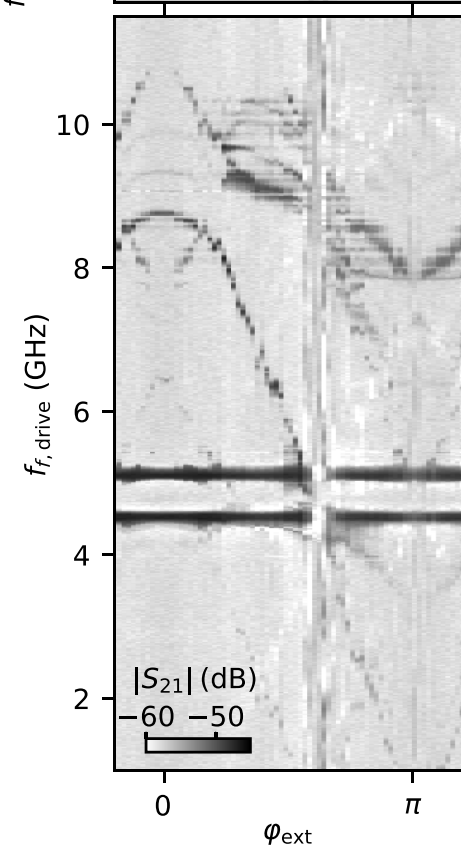

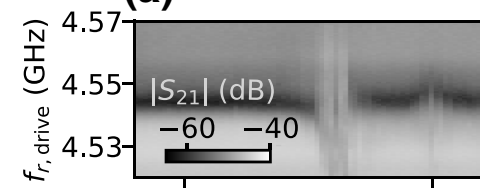

(b)

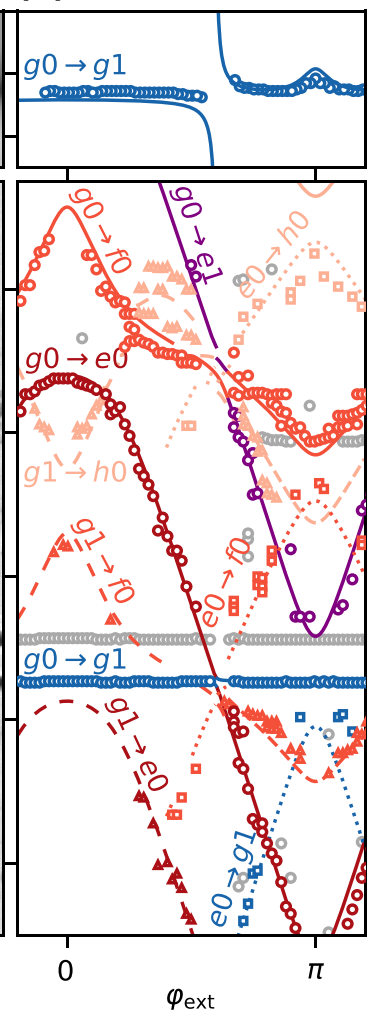

FIG. 2. Two-tone spectroscopy of device $A$, at $B_{z}=0$. (a) Magnitude of the transmitted readout signal as a function of the external flux and $f_{r \text {,drive }}$ (top) and $f_{f}$, drive (bottom), showing the flux modulation of the different transitions. (b) Extrema (maxima and minima) extracted from (a) (markers) and fitted transition frequencies (lines) obtained from the numerical diagonalization of the model Hamiltonian [Eq. (1)]. Gray markers indicate extrema that are not associated with any fluxoniumresonator transitions. A value of $E_{J} / h=6.7 \mathrm{GHz}$ is extracted from the fit.

$B_{x}$. Threading a flux quantum through the gradiometer corresponds to $B_{x}=550 \mu \mathrm{T}$, which is much greater than the $15 \mu \mathrm{T}$ that would be needed to thread a flux quantum through one of the two symmetric loops. The gradiometric geometry thus reduces the sensitivity of the fluxonium to magnetic field noise larger than the fluxonium loop by more than an order of magnitude.

To fit the spectroscopy data [markers in Fig. 2(b)], we diagonalize the Hamiltonian for the coupled fluxoniumresonator system shown in Fig. 1(a), $\hat{H}$ [46]. In the limit $L_{f} \gg L_{s}, L_{r}\left(\right.$ where $L_{f}=\Phi_{0}^{2} / 4 \pi^{2} E_{L}$ and $\left.\Phi_{0}=h / 2 e\right)$,

$\hat{H}=\frac{2 e^{2}}{C_{r}} \hat{n}_{r}^{2}+\frac{1}{2} \frac{\left(\Phi_{0} / 2 \pi\right)^{2}}{\left(L_{r}+L_{s}\right)} \hat{\varphi}_{r}^{2}-\frac{1}{2} \frac{\left(\Phi_{0} / 2 \pi\right)^{2} L_{s}}{L_{f}\left(L_{r}+L_{s}\right)} \hat{\varphi}_{r} \hat{\varphi}_{f}+\hat{H}_{f}$,
TABLE I. Device parameters obtained by fitting spectra in Figs. $2-4$ and by electromagnetic simulations. Here $h$ is Planck's constant.

\begin{tabular}{ccc}
\hline \hline & Device $A$ & Device $B$ \\
\hline$E_{C} / h(\mathrm{GHz})$ & 2.35 & 1.75 \\
$E_{L} / h(\mathrm{GHz})$ & 0.7 & 1.1 \\
$C_{r}(\mathrm{fF})$ & 26 & 26 \\
$L_{r}(\mathrm{nH})$ & 47 & 42 \\
$L_{s}(\mathrm{nH})$ & 8.5 & 4.6 \\
\hline \hline
\end{tabular}

where

$$
\hat{H}_{f}=4 E_{C} \hat{n}_{f}^{2}-E_{J}\left(V_{j}, B\right) \cos \left(\hat{\varphi}_{f}\right)+\frac{1}{2} E_{L}\left(\hat{\varphi}_{f}-\varphi_{\mathrm{ext}}\right)^{2}
$$

is the Hamiltonian of the uncoupled fluxonium. Here, $\hat{\varphi}_{f}$ and $\hat{\varphi}_{r}$ are the phase drops across the fluxonium junction and across $C_{r}$, respectively, and $\hat{n}_{f}$ and $\hat{n}_{r}$ are their conjugated charges. We leave all circuit parameters free except for $C_{r}=26 \mathrm{fF}$, which we extract from electromagnetic simulations. The parameters obtained from the fit are shown in Table I and the fitted transition frequencies are denoted with lines in Fig. 2(b). Each state is identified by the closest state in energy for the uncoupled system. In addition to transitions originating from the ground state, $g 0$, we also observe transitions for which the initial state is $g 1$, with one photon in the resonator (dashed lines). This is due to the continuous drive used to monitor $\left|S_{21}\right|$ at $f_{g 0 \rightarrow g 1}$, which can populate the resonator. Transitions starting from the first excited fluxonium state, $e 0$, around $\varphi_{\mathrm{ext}}=\pi$ (dotted lines) are also observed. The transition frequency for $g 0 \rightarrow e 0$ goes below $1 \mathrm{GHz}$ near $\varphi_{\mathrm{ext}}=\pi$. The transitions from $e 0$ thus occur due to the expected equilibrium thermal occupation of $e 0$ for temperatures of around $20 \mathrm{mK}$. We find excellent agreement between the experimental data and the fit, with all fit parameters coming to within 5\% of the designed circuit parameters except for $E_{J}$, which we can only coarsely predict. We have etched a $80 \mathrm{~nm}$ junction to maximize $E_{J}$, but the specific values of $E_{J}$ are determined by the interaction with the electrostatic gate and the microscopic details of the junction.

\section{ELECTROSTATIC TUNING OF FLUXONIUM PARAMETERS}

Mesoscopic phenomena often require fine tuning of the charge carrier density in the semiconductor. We here demonstrate that the spectrum of the fluxonium is measurable over a large range of gate voltages and thus does not limit the possible observable phenomena. We first measure $f_{g 0 \rightarrow g 1}$ vs $V_{j}$. As shown in Fig. 3(a), the resonator frequency is constant at low and high voltage values but has nonmonotonic fluctuations in an intermediate range, which is consistent with observations in previous experiments on nanowire junctions [4-6,29], where these fluctuations were 
(a)

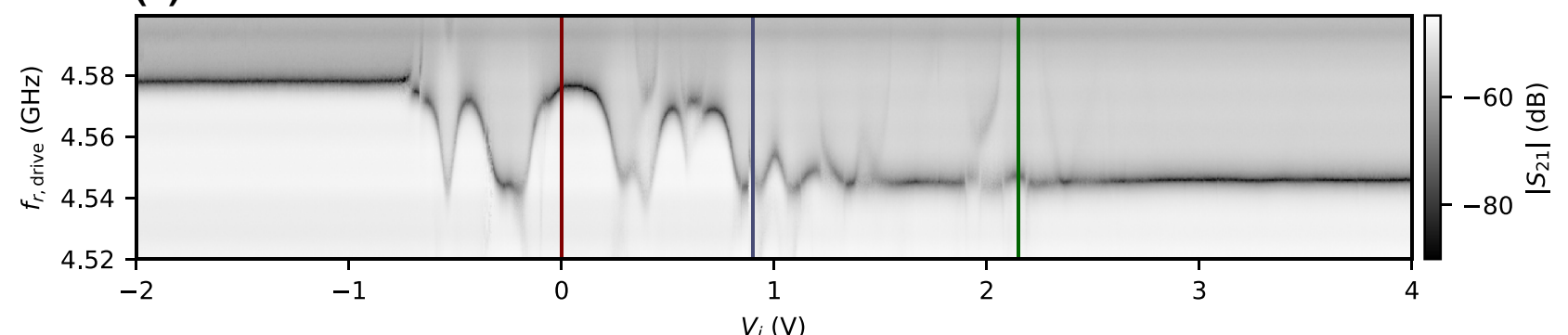

(b) (c)

(d)

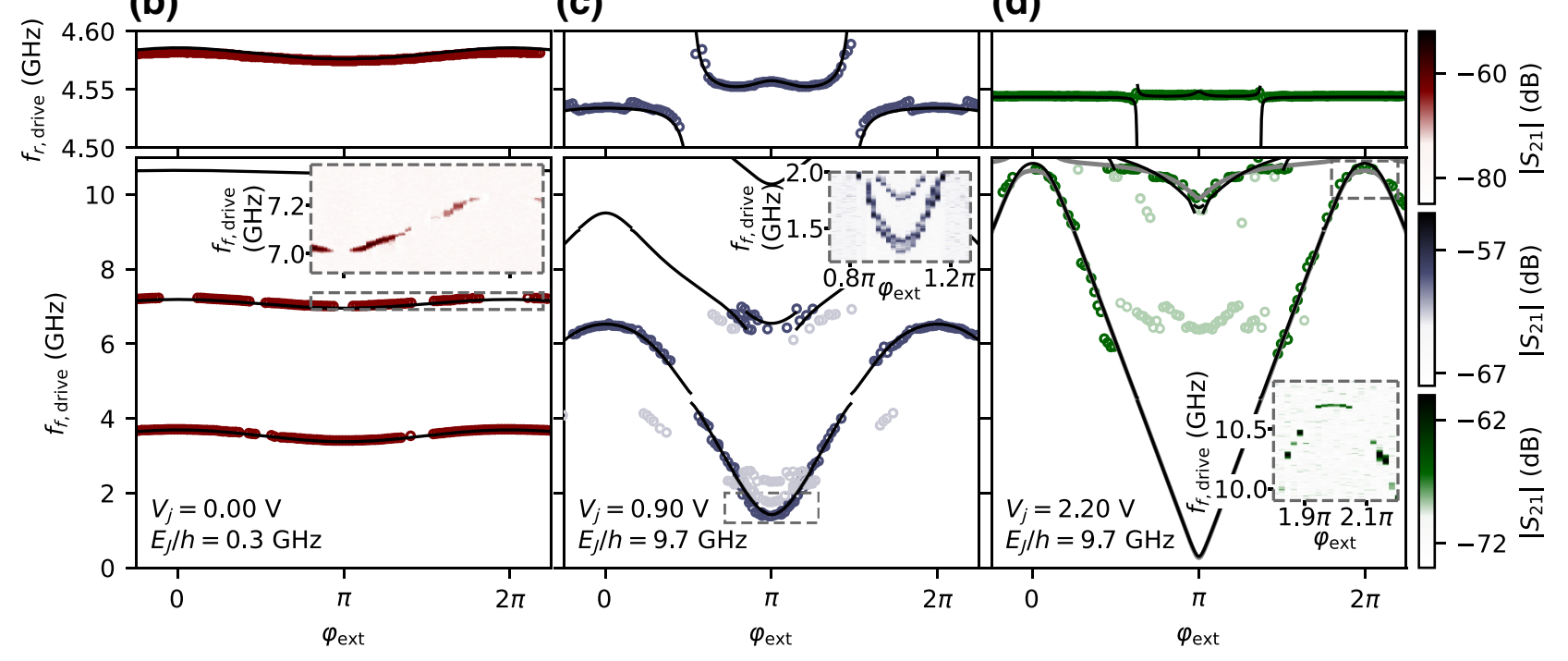

FIG. 3. Gate tuning of $E_{J}$ in device $A$, at $B_{z}=0$. (a) Gate dependence of the resonator's resonant frequency, $f_{g 0 \rightarrow g 1}$, at $\varphi_{\mathrm{ext}}=1.25 \pi$. (b)-(d) Fluxonium spectra at three different gate points, indicated with vertical lines in (a). The markers correspond to the peaks extracted from the measured resonator (top) and two-tone (bottom) transmission data. The fitted transition frequencies $f_{g 0 \rightarrow e 0}, f_{g 0 \rightarrow f 0}$, and $f_{g 0 \rightarrow h 0}$ (black lines) are obtained by fitting the darker markers with Hamiltonian (1). The values of $E_{J} / h$ extracted are $0.2,3.8$, and $9.6 \mathrm{GHz}$, respectively. For (d), the spectrum can be fitted more accurately with a Hamiltonian including one highly transparent channel (gray lines). The insets in (b)-(d) show sections of the measured transmission magnitude. In (b) we observe gaps in visibility at zero and half flux in the $g 0 \rightarrow f 0$ transition.

attributed to consecutive openings of different junction channels in the nanowire, whose transparencies oscillate with the gate. The behavior of the $f_{g 0 \rightarrow g 1}$ transition provides insight into the $V_{j}$ dependence of the fluxonium $E_{J}$. The value of $f_{g 0 \rightarrow g 1}$ can be seen as the bare resonant frequency of the uncoupled resonator plus a dispersive shift caused by the coupling to the fluxonium. The dispersive shift depends on the frequency of all level transitions of the coupled fluxonium-resonator system and is thus different for different values of $E_{J}$. This change in the dispersive shift leads to the observed changes in the measured resonant frequency of the resonator.

We now investigate directly the fluxonium spectrum. In Figs. 3(b)-3(d) we present spectra taken at three different $V_{j}$ values [marked by solid lines in Fig. 3(a)]. Here we use a lower drive power than in Fig. 2 to reduce broadening of the spectral lines due to the drive power; therefore, the main observable transitions start from the ground state, $g 0$. For low $V_{j}$, we observe a weakly anharmonic spectrum [Fig. 3(b)]. For large $V_{j}$ values [Figs. 3(c) and 3(d)], however, the flux dependence and the anharmonicity become much stronger. We also note the presence of additional transitions in the spectroscopy data for intermediate and large $V_{j}$ denoted by the lighter markers in Figs. 3(c) and $3(\mathrm{~d})$. The spectrum at intermediate $V_{j}$ are taken at a point where the junction is very sensitive to the gate voltage; the additional transitions are due to the $E_{J}$ of the junction fluctuating while the spectroscopy is being performed. At higher $V_{j}$, the $E_{J}$ of the junction is stable as a function of the gate but additional transitions due to the presence of the resonator drive are also observed. We fit the data of the three spectra using the same parameters as those used in Fig. 2 while only allowing $E_{J}$ to vary. The fits maintain their accuracy over the whole $V_{j}$ range. We do note, however, that, for large $E_{J}$, the fit starts to deviate from the data for transitions to higher excited states [Fig. 3(d)]. This may 
be due to a nonsinusoidal energy-phase relation of a more transparent channel in the junction. A fit using a Hamiltonian including the Andreev potential for a short transparent junction instead of the standard $\cos \varphi$ potential is shown in gray in Fig. 3(d). Details of the fit as well as additional fits to the data in Figs. 3(b) and 3(c) using the Andreev potential in the Hamiltonian can be found in the Appendix.

We have therefore shown that the behavior of a hybrid fluxonium circuit with a semiconducting weak link can still be predicted and understood using a simple Hamiltonian with a few degrees of freedom. Moreover, we can conclude that $E_{J}$ is the only circuit parameter affected by large changes in $V_{j}$ and that it generally increases with $V_{j}$. Our results thus show that it is possible to address and observe the state of the system over a large $E_{J}$ range encompassing regimes where its eigenstates are of very different character.

(a)
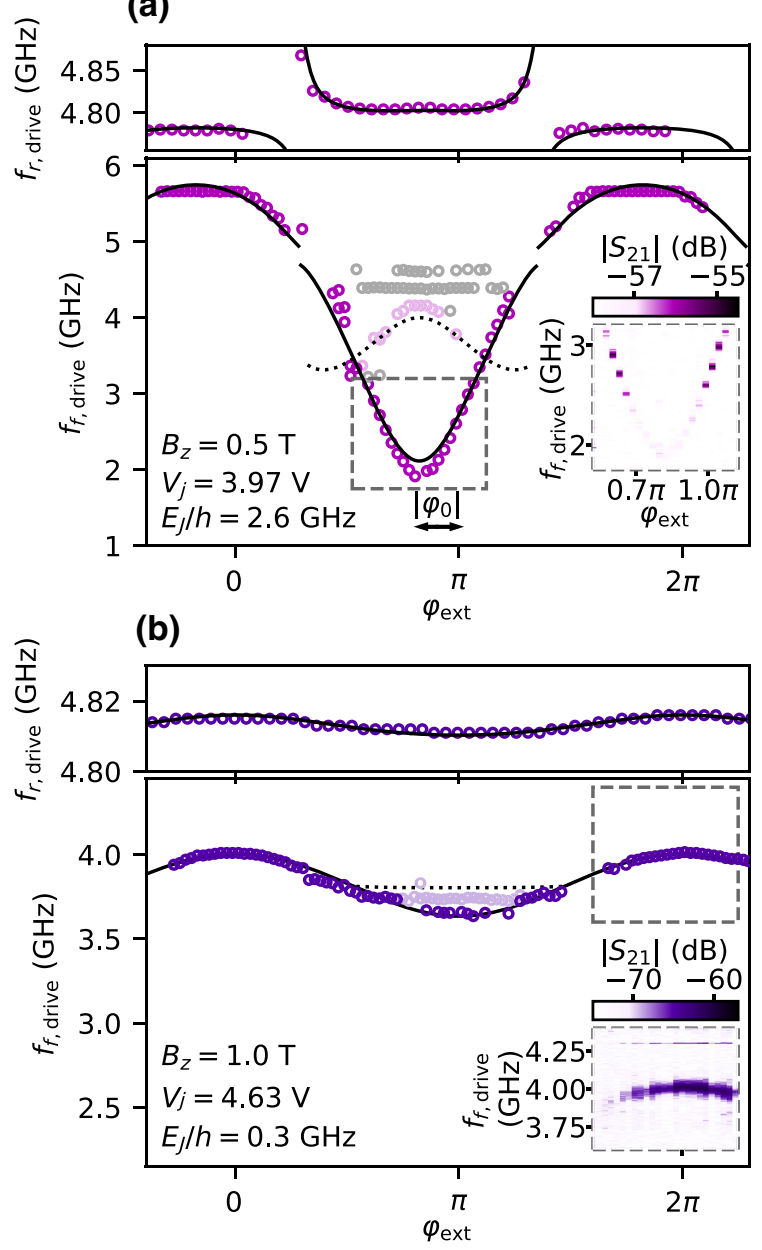

\section{FLUXONIUM BEHAVIOR IN A MAGNETIC FIELD}

Next, we explore the magnetic field compatibility of the nanowire fluxonium. The magnetic field behavior of the device strongly depends on the microscopic details of the nanowire junction. In order to demonstrate the field compatibility of the fluxonium circuit elements, we here show data from device $B$ whose parameters are optimized for magnetic field compatibility. The magnetic field behavior of device $A$ is provided in the Supplemental Material [47]. Spectroscopy measurements at two different $V_{j}$ and $B_{z}$ points are shown in Figs. 4(a) and 4(b). We continue to be able to perform spectroscopy on the fluxonium over the full range in $\varphi_{\text {ext }}$ at fields up to $1 \mathrm{~T}$. We do note, however, that at higher magnetic fields, the thermal occupation of the excited state of the fluxonium does increase since we observe transitions from this state even when the $g 0 \rightarrow e 0$

(c)

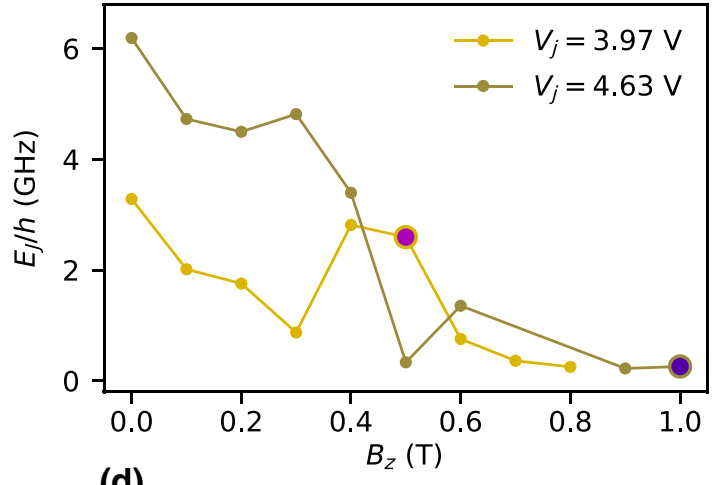

(d)

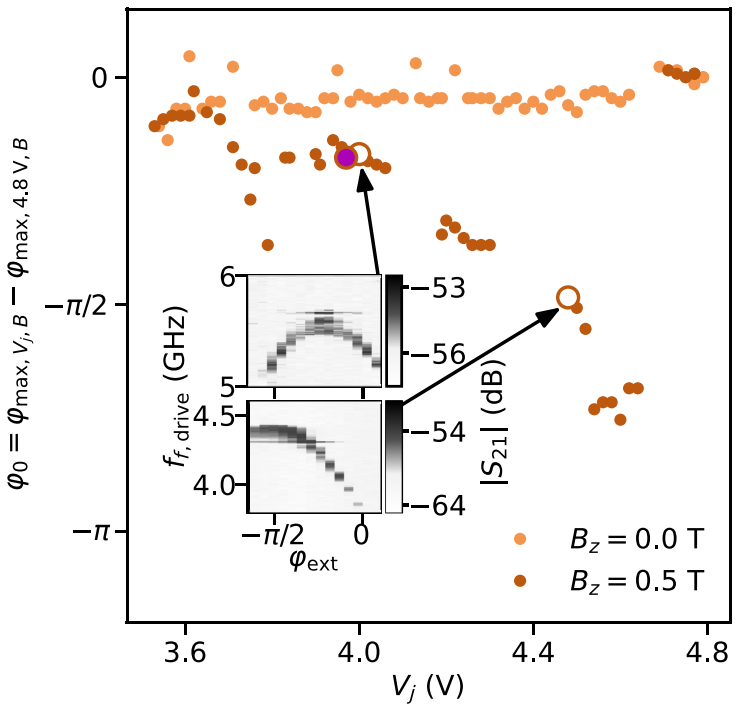

FIG. 4. Behavior of device $B$ in a magnetic field. (a),(b) Fluxonium spectra at two different $V_{j}$ and $B_{z}$ points. The $E_{J}$ value extracted from the fit is lower at higher magnetic fields. In (a) we observe a $\varphi_{0}=-0.16 \pi$ phase shift with respect to a reference $\varphi_{\text {ext }}$ taken at the same field at $V_{j}=4.80 \mathrm{~V}$. (c) Plot of $E_{J}$ vs $B_{z}$ at two different gate voltage points. The $E_{J}$ value decreases nonmonotonically with the field. (d) Plot of $\varphi_{0}$ vs $V_{j}$ at two different magnetic field points. At $B_{z}=0.0 \mathrm{~T}, \varphi_{0}$ stays constant for the whole $V_{j}$ range. At $B_{z}=0.5 \mathrm{~T}$, however, there is a continuous $\varphi_{0}$ shift that ranges from 0 to $-\pi$. The two insets show the zero-flux spectroscopy feature shifted from zero at two different gate points. In (c),(d) the points corresponding to the spectra in (a),(b) are highlighted with matching colors. 
frequency is above $1 \mathrm{GHz}$. Importantly, we can still fit the spectroscopy data accurately in this regime, indicating that the fluxonium-resonator Hamiltonian remains valid at high magnetic fields, with $E_{J}$ being the only parameter largely affected by $B_{z}$. Fit parameters for device $B$ are shown in Table I.

We finally use the nanowire fluxonium to investigate the behavior of spin-orbit-coupled semiconducting junctions in a magnetic field. We perform spectroscopy measurements at gate voltages ranging from 3.5 to $4.8 \mathrm{~V}$ and fields ranging from 0 to $1 \mathrm{~T}$. From the spectroscopy we extract $E_{J}$ as a function of $B_{z}$ at two different gate points, which is shown in Fig. 4(c). A nonmonotonic decrease of $E_{J}$ with the field is observed at both gate points. We expect an overall decrease in $E_{J}$ vs $B_{z}$ due to the superconducting gap closing at high magnetic fields. The nonmonotonic behavior of $E_{J}$, however, suggests the presence of interference between different modes in the junction [27]. Additional in-field $E_{J}$ data is provided in Sec. V of the Supplemental Material [47]. An overall decrease of $E_{J}$ with $B_{z}$ is observed for all investigated gate values, while the nonmonotonic dependence is often, but not always, observed.

A shifting of the zero-flux point in the spectroscopy of the fluxonium device at high fields can be used to determine the breaking of multiple symmetries in the semiconducting junction [48]. This phase shift is known as the $\varphi_{0}$-Josephson effect, which occurs when chiral and time-reversal symmetries are both broken in the junction. In InSb- and InAs-based junctions, this symmetry breaking originates from the interplay between the presence of multiple channels in the junction, spin-orbit coupling, and the Zeeman splitting due to the applied magnetic field $[29,48,49]$. We observe such a shift in the zero-flux point of the spectroscopy lines as $V_{j}$ is varied in a $B_{z}$ field [indicated by $\varphi_{0}=\varphi_{\max , V_{i}, B}-\varphi_{\max , 4.8 V, B}$ in Fig. 4(a)]. The $\varphi_{0}$ shift as a function of $V_{j}$ is shown, at $B_{z}=0$ and at $B_{z}=0.5 \mathrm{~T}$, in Fig. 4(d). The $\varphi_{0}$ value at $V_{j}=4.80 \mathrm{~V}$ is taken as the $\varphi_{0}=0$ reference at each $B_{z}$. The value of $\varphi_{0}$ is thus a relative value at each $B_{z}$. At $B_{z}=0$ the zero-flux point does not change, while it changes continuously with $V_{j}$ when a $B_{z}$ field is applied. Since the observed phase shift appears as a function of only $V_{j}$ at fixed magnetic fields, we can exclude trivial effects such as misalignment of the magnetic field. We note that we observe shifts in $\varphi_{0}$ approaching $\pi$, which is significantly larger than previously predicted [48]. Mixing between a large number of spin-split Andreev channels in the junction may lead to the larger observed shifts. Additional $\varphi_{0}$ data as a function of $V_{j}$ and $B$ can be found in the Supplemental Material [47].

\section{SPECTROSCOPIC LINEWIDTH}

For the fluxonium's applications as a detector of field-dependent mesoscopic effects, its spectral lines must be measurable under the magnetic field and electrostatic conditions of interest, but also must maintain a narrow linewidth in all regimes. The spectroscopic linewidth bounds the resolution with which we can resolve the splitting of lines due to, for example, quasiparticle poisoning of highly transparent ABSs or MZMs at the junction.

We have performed a spectroscopic linewidth analysis for devices $A$ and $B$ at different points in external flux (Fig. 5): near the flux sweet spot at $\varphi_{\text {ext }}=0$ and also at $\varphi_{\text {ext }}=0.3 \pi$. As shown in Fig. 5(a), the linewidth of device $B$ stays below $50 \mathrm{MHz}$ up to $1 \mathrm{~T}$ at the $\varphi_{\mathrm{ext}}=0$. However, the linewidth overall becomes larger, for both
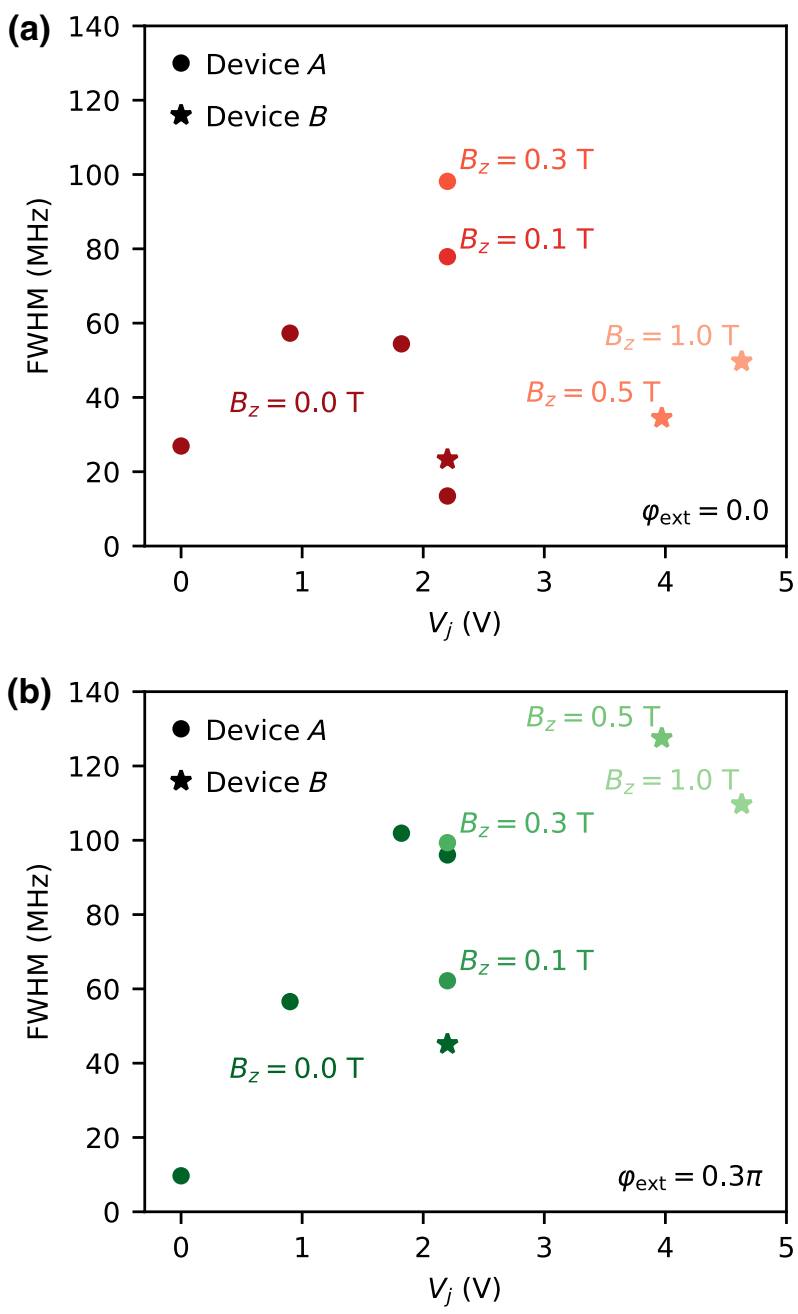

FIG. 5. Spectroscopic linewidth. Extracted linewidth [full width at half maximum (FWHM)] of the $g \rightarrow e$ transition as a function of $V_{j}$ for device $A$ (circular markers) and device $B$ (star markers). Different color strengths denote different magnetic fields. The data for device $A$ correspond to the spectra shown in Figs. 3(b)-3(d) and Fig. 2 of the Supplemental Material [47]. The in-field data for device $B$ correspond to the spectra shown in Figs. 4(a) and 4(b). The linewidths are extracted in symmetric $0.05 \pi$ flux windows around $\varphi_{\mathrm{ext}}=0$, which is a sweet spot for the external flux, and $\varphi_{\text {ext }}=0.3 \pi$ for (a),(b), respectively. 
devices, at $\varphi_{\text {ext }}=0.3 \pi$ [Fig. 5(b)]. This behavior is consistent with residual flux noise broadening the linewidth of the fluxonium. Our linewidth analysis indicates that the energy resolution of the fluxonium would, if measuring near $\varphi_{\text {ext }}=0$, be at most $0.4 \mu \mathrm{eV}$ in all regimes, which significantly improves upon the resolution that can currently be reached by transport experiments where the linewidth is limited by thermal broadening (typically approximately $10 \mu \mathrm{eV})$.

We do note that in our experiment the fluxonium and resonator parameters are chosen for the greatest visibility over the largest range of $E_{J}$ and $\varphi_{\text {ext }}$. Improvements upon the design, such as weaker coupling to the readout resonator, smaller asymmetry in the gradiometric superinductance, and materials optimizations such as changing the dielectric of the fluxonium capacitor, would lead to significantly narrower linewidths.

\section{CONCLUSION}

In conclusion, we have successfully realized a gatetunable fluxonium resilient to high magnetic fields. We have combined a gate-controlled junction with magneticfield-compatible materials and a gradiometric design to build the hybrid fluxonium. We are able to perform spectroscopy over a large range of gate voltages and in-plane magnetic fields. We have used the fluxonium to investigate the behavior of an InAs Josephson junction in a magnetic field and observed a nonmonotonic decrease of the $E_{J}$ of the junction as well as the $\varphi_{0}$-Josephson effect. The observed $\varphi_{0}$ shift is gate tunable up to $\varphi_{0}=\pi$. One could therefore use this effect to build extremely small, flux-noise insensitive superconducting circuits where the phase difference of the junction could be tuned using a gate voltage.

The magnetic-field-compatible hybrid fluxonium is also now ready to detect the $4 \pi$-periodic Josephson effect [25] and measure Majorana parity dynamics. We remark that, while our experiment was performed with similar materials and in similar magnetic fields to previous experiments where transport signatures pointed to the presence of MZMs, we did not observe any signatures of MZMs. Recent theoretical work has suggested that previously observed signatures of MZMs may be due to trivial Andreev bound states [50] and has advocated for cleaner materials with stronger spin-orbit coupling proximitized by superconductors with larger energy gaps. Future experiments will thus incorporate InSb nanowires with thinner Al shells as the small junction of the hybrid fluxonium.

The excellent agreement we have demonstrated between data and theory indicates that one can engineer hybrid circuits such that their behavior can continue to be understood even when the circuit is complicated by the interplay of superconductivity, spin-orbit coupling, and magnetism.
This opens avenues for using the hybrid fluxonium to explore superconductivity in the presence of a magnetic field as well as reading out and controlling material platforms that require the application of a large magnetic field. Since it is straightforward to incorporate different materials into the hybrid fluxonium, proposals to probe superconductor-proximitized edge states in a quantum spin Hall insulator [51,52] or field-dependent spin-polarized correlated insulating phases $[53,54]$ are now possible using our hybrid circuit.

\section{ACKNOWLEDGMENTS}

We thank W. Uilhoorn for fabrication advice. We also thank B. van Heck and A. Antipov for helpful discussions. This research was cofunded by the allowance for Top consortia for Knowledge and Innovation (TKI) from the Dutch Ministry of Economic Affairs and the Microsoft Quantum initiative.

\section{APPENDIX: MODELING THE JUNCTION ACCOUNTING FOR HIGHLY TRANSPARENT TRANSMISSION CHANNELS}

In Figs. 2-4 we fit the spectroscopy data assuming a sinusoidal current-phase relation at the junction, which results in the term $-E_{J} \cos (\varphi)$ in the Hamiltonian [Eq. (2)], which is characteristic of $S-I-S$ junctions with low-transparency channels. A more accurate model for the potential of a semiconducting Josephson junction with $N$ channels with transparencies $T_{i}$ is [55]

$$
V_{\mathrm{SJJ}}(\varphi)=\Delta \sum_{i=1}^{N} \sqrt{1-T_{i} \sin ^{2}(\varphi / 2)},
$$

where $\Delta$ denotes the induced gap on the proximitized sections of the nanowire. When the transparencies of the different channels are low, this dependence converges to a sinusoidal relation with $E_{J}=\Delta \sum_{i=1}^{N} T_{i} / 4$.

Here, we show fits to the spectroscopy data from Fig. 3 using the semiconducting junction potential $V_{\text {SJJ }}$ with a different number of channels and for various values of $\Delta$. In Fig. 6 we show fits to the transitions starting from the ground state with the Hamiltonian for the uncoupled fluxonium [Eq. (2)] using a sinusoidal potential, a onechannel potential, and a two-channel potential. In Fig. 7 we show the $\Delta$ sensitivity of the one-channel spectrum, showing the fit accuracy for $\Delta$ values lower and higher than the optimum value. For low and intermediate $E_{J}$ values, the accuracy of the different models is very similar. For high $E_{J}$, however, the fits to the high-frequency transitions are more accurate when highly transparent channels are included. This points toward the presence of at least one highly transparent channel in the junction. The fit accuracy is however similar when one, two, or more channels 
(a)

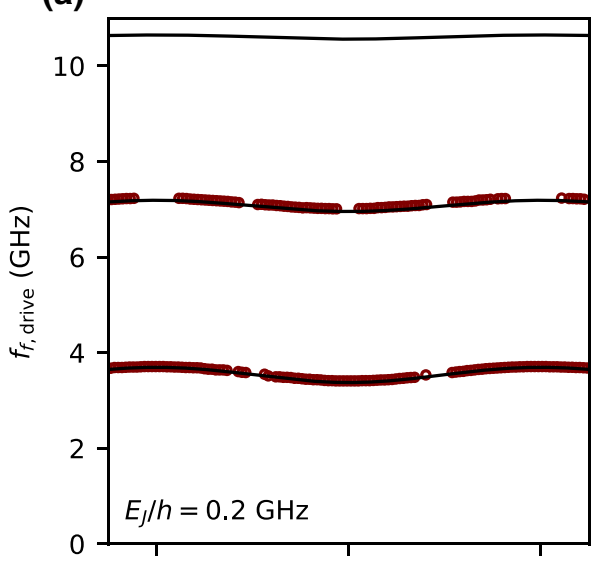

(d)

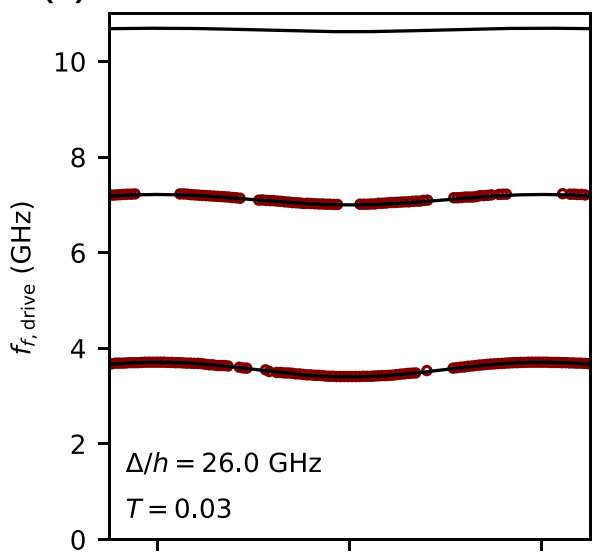

(g)

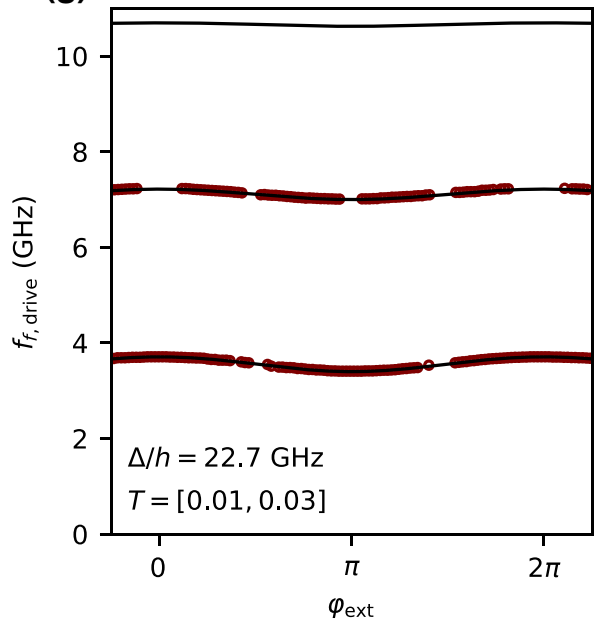

(b)

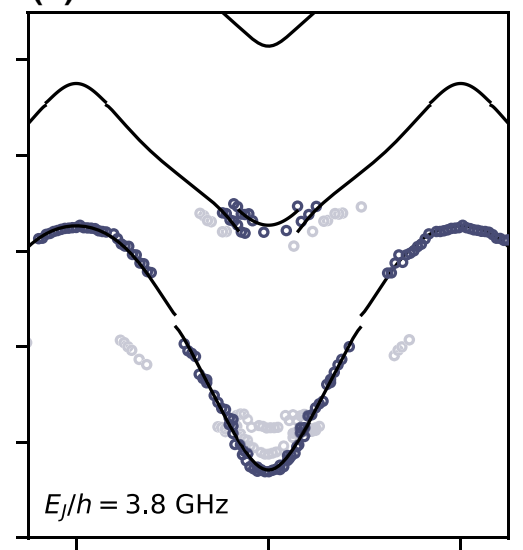

(e)

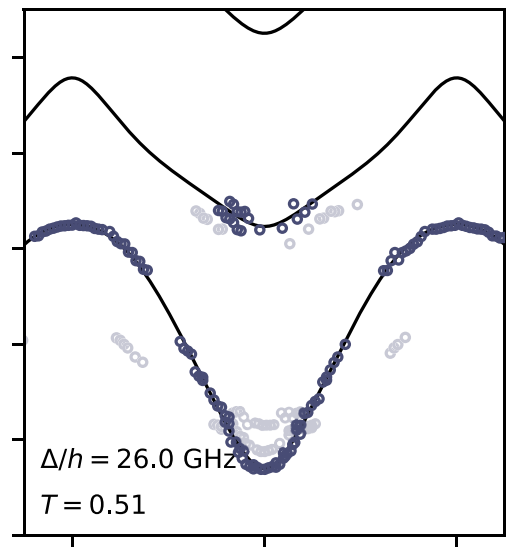

(h)

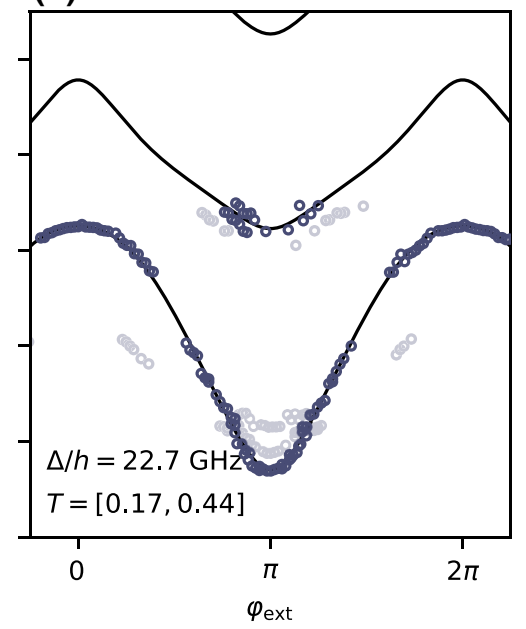

(c)

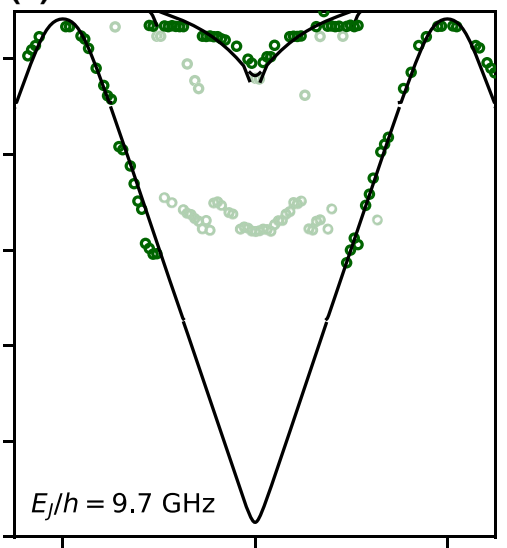

(f)

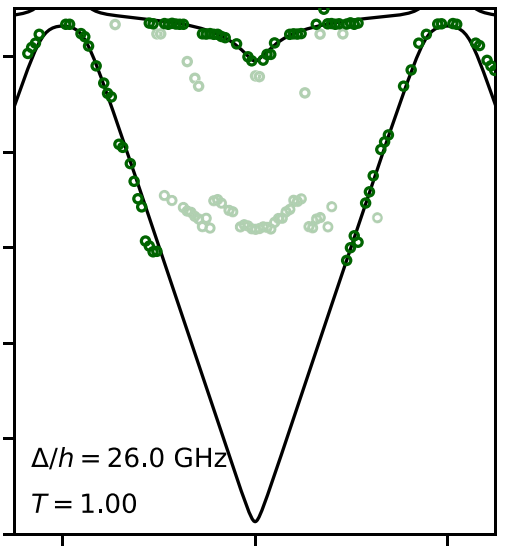

(i)

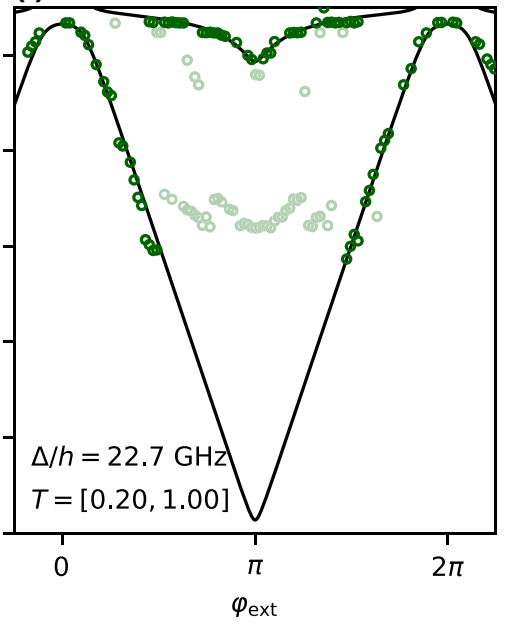

FIG. 6. Fits using the Andreev potential in the Hamiltonian. Spectroscopy data fit with the uncoupled fluxonium Hamiltonian [Eq. (2)] with a sinusoidal potential (first row), with a one-channel potential (second row), and with a two-channel potential (third row). Only the dark markers, corresponding to transitions starting from the ground state, are included in the fit. We obtain $E_{C} / h=2.35$ $\mathrm{GHz}$ and $E_{L} / h=0.7 \mathrm{GHz}$ for the three different fits. The rest of the fit parameters are indicated in the different panels.

are considered. We thus cannot extract a measure of the number of junction channels by performing fits of the spectroscopic data.

We furthermore observe that the best fit assuming a single channel occurs at $\Delta=26 \mathrm{GHz}$. This is smaller than the gap $(\Delta=53 \mathrm{GHz})$ typically measured in transport experiments. This discrepancy may be due to the necessity of incorporating excited Andreev states into the fluxonium model or interactions due to an accidental quantum dot in the junction $[42,56,57]$. 
(a)

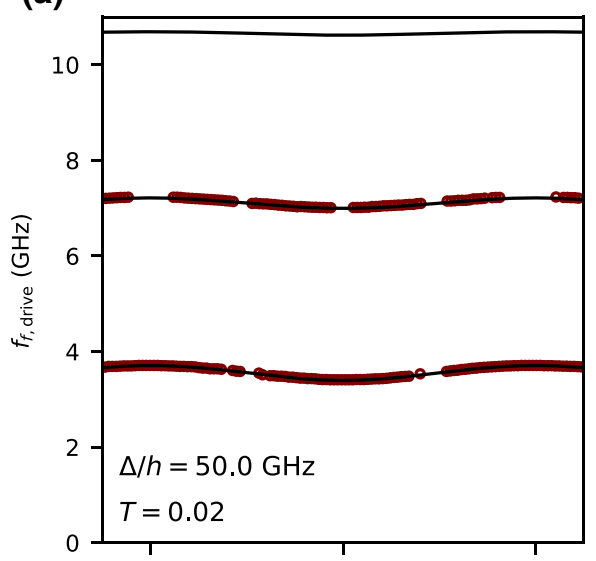

(d)

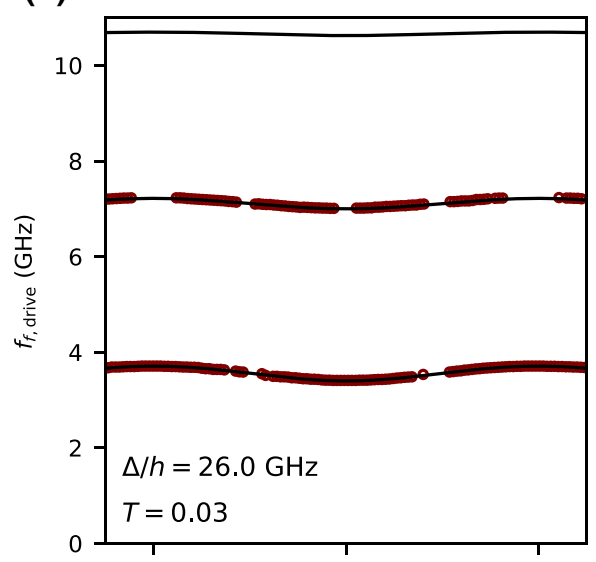

(g)

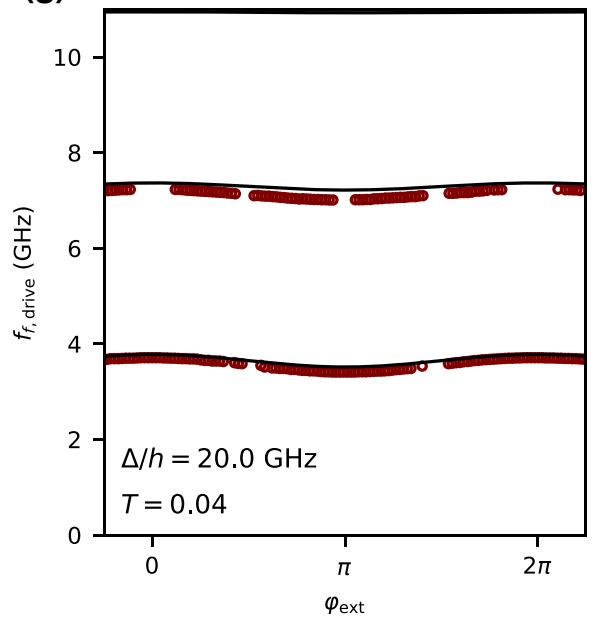

(b)

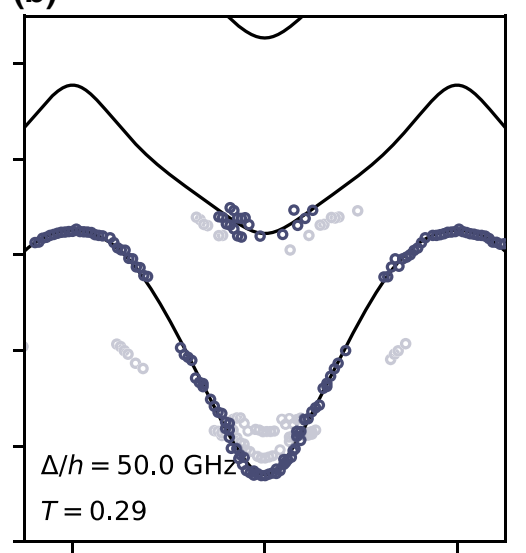

(e)

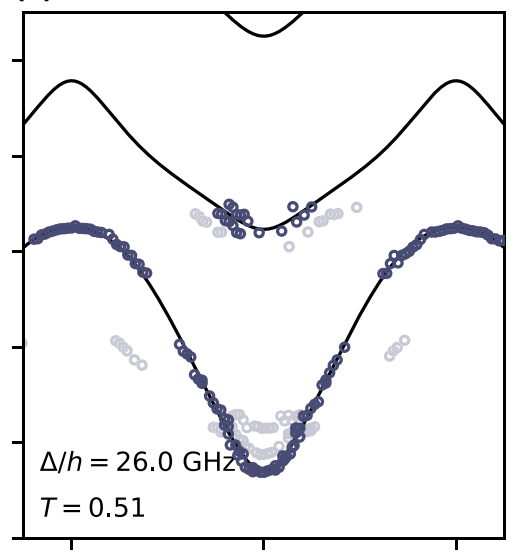

(h)

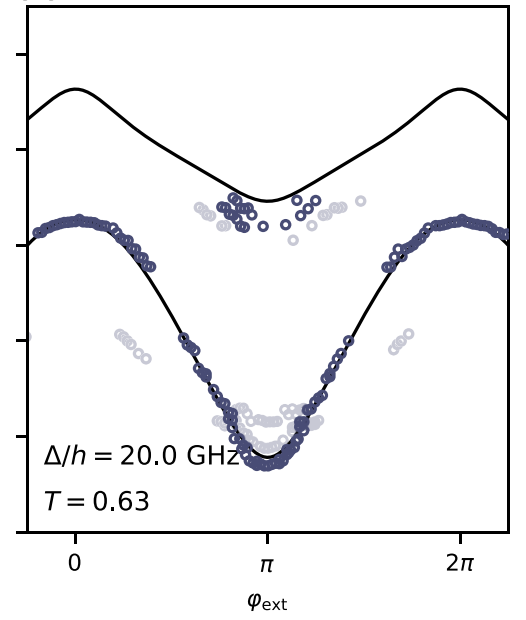

(c)

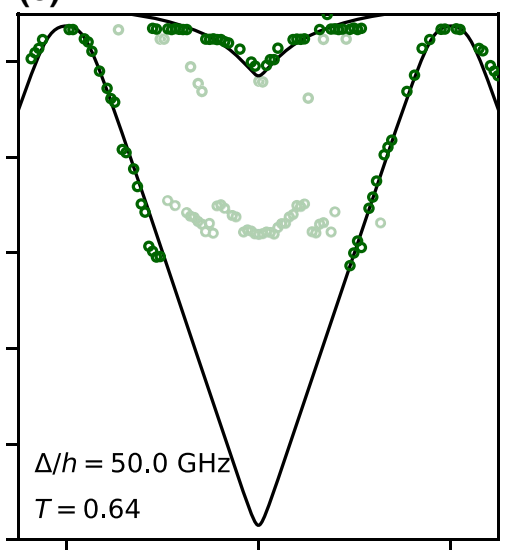

(f)

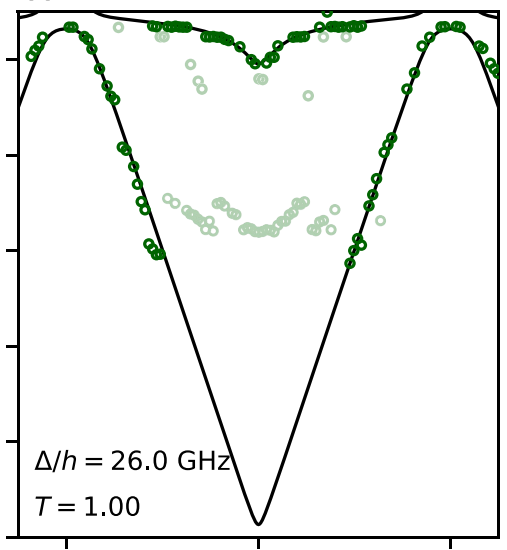

(i)

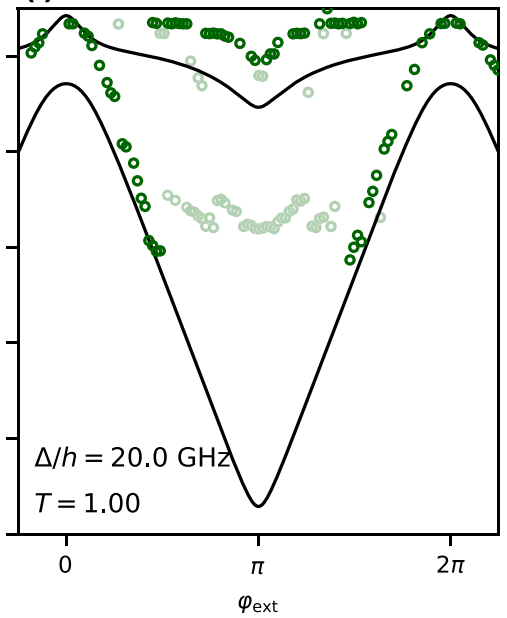

FIG. 7. The $\Delta$ dependence of fits using the Andreev potential in the Hamiltonian. Spectroscopy data fitted with the uncoupled fluxonium Hamiltonian [Eq. (2)] with a one-channel potential. In the first and third rows the value of $\Delta$ is fixed to $\Delta / h=50.0 \mathrm{GHz}$ and $\Delta / h=20.0 \mathrm{GHz}$, respectively. In the second row $\Delta$ is left free and $\Delta / h=26.0 \mathrm{GHz}$ is obtained as the optimum value. Only the dark markers, corresponding to transitions starting from the ground state are included in the fit.

[1] A. Blais, R.-S. Huang, A. Wallraff, S. M. Girvin, and R. J. Schoelkopf, Cavity quantum electrodynamics for superconducting electrical circuits: An architecture for quantum computation, Phys. Rev. A 69, 062320 (2004).
[2] I. Chiorescu, P. Bertet, K. Semba, Y. Nakamura, C. J. P. M. Harmans, and J. E. Mooij, Coherent dynamics of a flux qubit coupled to a harmonic oscillator, Nature 431, 159 (2004).

[3] A. Wallraff, D. I. Schuster, A. Blais, L. Frunzio, R.-S. Huang, J. Majer, S. Kumar, S. M. Girvin, and 
R. J. Schoelkopf, Strong coupling of a single photon to a superconducting qubit using circuit quantum electrodynamics, Nature 431, 162 (2004).

[4] G. de Lange, B. van Heck, A. Bruno, D. J. van Woerkom, A. Geresdi, S. R. Plissard, E. P. A. M. Bakkers, A. R. Akhmerov, and L. DiCarlo, Realization of Microwave Quantum Circuits Using Hybrid Superconducting-Semiconducting Nanowire Josephson Elements, Phys. Rev. Lett. 115, 127002 (2015).

[5] T. W. Larsen, K. D. Petersson, F. Kuemmeth, T. S. Jespersen, P. Krogstrup, J. Nygård, and C. M. Marcus, Semiconductor-Nanowire-Based Superconducting Qubit, Phys. Rev. Lett. 115, 127001 (2015).

[6] F. Luthi, T. Stavenga, O. W. Enzing, A. Bruno, C. Dickel, N. K. Langford, M. A. Rol, T. S. Jespersen, J. Nygård, P. Krogstrup, and L. DiCarlo, Evolution of Nanowire Transmon Qubits and Their Coherence in a Magnetic Field, Phys. Rev. Lett. 120, 100502 (2018).

[7] M. Hays, G. de Lange, K. Serniak, D. J. van Woerkom, D. Bouman, P. Krogstrup, J. Nygård, A. Geresdi, and M. H. Devoret, Direct Microwave Measurement of AndreevBound-State Dynamics in a Semiconductor-Nanowire Josephson Junction, Phys. Rev. Lett. 121, 047001 (2018).

[8] X. Mi, J. V. Cady, D. M. Zajac, P. W. Deelman, and J. R. Petta, Strong coupling of a single electron in silicon to a microwave photon, Science 355, 156 (2017).

[9] L. Casparis, M. R. Connolly, M. Kjaergaard, N. J. Pearson, A. Kringhøj, T. W. Larsen, F. Kuemmeth, T. Wang, C. Thomas, S. Gronin, G. C. Gardner, M. J. Manfra, C. M. Marcus, and K. D. Petersson, Superconducting gatemon qubit based on a proximitized two-dimensional electron gas, Nat. Nanotechnol. 13, 915 (2018).

[10] J. G. Kroll, W. Uilhoorn, K. L. van der Enden, D. de Jong, K. Watanabe, T. Taniguchi, S. Goswami, M. C. Cassidy, and L. P. Kouwenhoven, Magnetic field compatible circuit quantum electrodynamics with graphene Josephson junctions, Nat. Commun. 9, 4615 (2018).

[11] J. I.-J. Wang, D. Rodan-Legrain, L. Bretheau, D. L. Campbell, B. Kannan, D. Kim, M. Kjaergaard, P. Krantz, G. O. Samach, F. Yan, J. L. Yoder, K. Watanabe, T. Taniguchi, T. P. Orlando, S. Gustavsson, P. Jarillo-Herrero, and W. D. Oliver, Coherent control of a hybrid superconducting circuit made with graphene-based van der waals heterostructures, Nat. Nanotechnol. 14, 120 (2019).

[12] M. Hays, V. Fatemi, K. Serniak, D. Bouman, S. Diamond, G. de Lange, P. Krogstrup, J. Nygård, A. Geresdi, and M. H. Devoret, Continuous monitoring of a trapped superconducting spin, Nat. Phys. 16, 1103 (2020).

[13] A. Zazunov, A. Brunetti, A. L. Yeyati, and R. Egger, Quasiparticle trapping, Andreev level population dynamics, and charge imbalance in superconducting weak links, Phys. Rev. B 90, 104508 (2014).

[14] J. I. Väyrynen, G. Rastelli, W. Belzig, and L. I. Glazman, Microwave signatures of Majorana states in a topological Josephson junction, Phys. Rev. B 92, 134508 (2015).

[15] B. van Heck, J. I. Väyrynen, and L. I. Glazman, Zeeman and spin-orbit effects in the Andreev spectra of nanowire junctions, Phys. Rev. B 96, 075404 (2017).

[16] K. Yavilberg, E. Ginossar, and E. Grosfeld, Differentiating majorana from andreev bound states in a superconducting circuit, Phys. Rev. B 100, 241408 (2019).
[17] F. Hassler, A. R. Akhmerov, and C. W. J. Beenakker, The top-transmon: A hybrid superconducting qubit for parityprotected quantum computation, New J. Phys. 13, 095004 (2011).

[18] T. Hyart, B. van Heck, I. C. Fulga, M. Burrello, A. R. Akhmerov, and C. W. J. Beenakker, Flux-controlled quantum computation with Majorana fermions, Phys. Rev. B 88, 035121 (2013).

[19] E. Ginossar and E. Grosfeld, Microwave transitions as a signature of coherent parity mixing effects in the Majoranatransmon qubit, Nat. Commun. 5, 4772 (2014).

[20] J. P. T. Stenger, M. Hatridge, S. M. Frolov, and D. Pekker, Braiding quantum circuit based on the $4 \pi$ Josephson effect, Phys. Rev. B 99, 035307 (2019).

[21] A. Y. Kitaev, Unpaired majorana fermions in quantum wires, Phys.-Uspekhi 44, 131 (2001).

[22] R. M. Lutchyn, E. P. A. M. Bakkers, L. P. Kouwenhoven, P. Krogstrup, C. M. Marcus, and Y. Oreg, Majorana zero modes in superconductor-semiconductor heterostructures, Nat. Rev. Mater. 3, 52 (2018).

[23] M. Sato and Y. Ando, Topological superconductors: A review, Rep. Prog. Phys. 80, 076501 (2017).

[24] S. D. Sarma, M. Freedman, and C. Nayak, Majorana zero modes and topological quantum computation, Npj Quantum Inf. 1, 15001 (2015).

[25] D. Pekker, C.-Y. Hou, V. E. Manucharyan, and E. Demler, Proposal for Coherent Coupling of Majorana Zero Modes and Superconducting Qubits Using the $4 \pi$ Josephson Effect, Phys. Rev. Lett. 111, 107007 (2013).

[26] J. Paajaste, M. Amado, S. Roddaro, F. S. Bergeret, D. Ercolani, L. Sorba, and F. Giazotto, Pb/inas nanowire josephson junction with high critical current and magnetic flux focusing, Nano Lett. 15, 1803 (2015).

[27] K. Zuo, V. Mourik, D. B. Szombati, B. Nijholt, D. J. van Woerkom, A. Geresdi, J. Chen, V. P. Ostroukh, A. R. Akhmerov, S. R. Plissard, D. Car, E. P. A. M. Bakkers, D. I. Pikulin, L. P. Kouwenhoven, and S. M. Frolov, Supercurrent Interference in Few-Mode Nanowire Josephson Junctions, Phys. Rev. Lett. 119, 187704 (2017).

[28] S. Hart, Z. Cui, G. Ménard, M. Deng, A. E. Antipov, R. M. Lutchyn, P. Krogstrup, C. M. Marcus, and K. A. Moler, Current-phase relations of inas nanowire josephson junctions: From interacting to multimode regimes, Phys. Rev. B 100, 064523 (2019).

[29] D. J. van Woerkom, A. Proutski, B. van Heck, D. Bouman, J. I. Väyrynen, L. I. Glazman, P. Krogstrup, J. Nygård, L. P. Kouwenhoven, and A. Geresdi, Microwave spectroscopy of spinful Andreev bound states in ballistic semiconductor Josephson junctions, Nat. Phys. 13, 876 (2017).

[30] L. Tosi, C. Metzger, M. F. Goffman, C. Urbina, H. Pothier, S. Park, A. L. Yeyati, J. Nygård, and P. Krogstrup, SpinOrbit Splitting of Andreev States Revealed by Microwave Spectroscopy, Phys. Rev. X 9, 011010 (2019).

[31] C. Janvier, L. Tosi, L. Bretheau, Ç. Ö. Girit, M. Stern, P. Bertet, P. Joyez, D. Vion, D. Esteve, M. F. Goffman, H. Pothier, and C. Urbina, Coherent manipulation of andreev states in superconducting atomic contacts, Science 349, 1199 (2015).

[32] P. Krogstrup, N. L. B. Ziino, W. Chang, S. M. Albrecht, M. H. Madsen, E. Johnson, J. Nygård, C. M. Marcus, and 
T. S. Jespersen, Epitaxy of semiconductor-superconductor nanowires, Nat. Mater. 14, 400 (2015).

[33] V. E. Manucharyan, Ph.D. Thesis, School Yale University, 2012.

[34] V. E. Manucharyan, J. Koch, L. I. Glazman, and M. H. Devoret, Fluxonium: Single Cooper-pair circuit free of charge offsets, Science 326, 113 (2009).

[35] K. Kalashnikov, W. T. Hsieh, W. Zhang, W.-S. Lu, P. Kamenov, A. Di Paolo, A. Blais, M. E. Gershenson, and M. Bell, Bifluxon: Fluxon-parity-protected superconducting qubit, PRX Quantum 1, 010307 (2020).

[36] H. Rotzinger, S. T. Skacel, M. Pfirrmann, J. N. Voss, J. Münzberg, S. Probst, P. Bushev, M. P. Weides, A. V. Ustinov, and J. E. Mooij, Aluminium-oxide wires for superconducting high kinetic inductance circuits, Superconductor Sci. Technol. 30, 025002 (2016).

[37] L. Grünhaupt, N. Maleeva, S. T. Skacel, M. Calvo, F. LevyBertrand, A. V. Ustinov, H. Rotzinger, A. Monfardini, G. Catelani, and I. M. Pop, Loss Mechanisms and Quasiparticle Dynamics in Superconducting Microwave Resonators Made of Thin-Film Granular Aluminum, Phys. Rev. Lett. 121, 117001 (2018).

[38] N. Maleeva, L. Grünhaupt, T. Klein, F. Levy-Bertrand, O. Dupre, M. Calvo, F. Valenti, P. Winkel, F. Friedrich, W. Wernsdorfer, A. V. Ustinov, H. Rotzinger, A. Monfardini, M. V. Fistul, and I. M. Pop, Circuit quantum electrodynamics of granular aluminum resonators, Nat. Commun. 9, 3889 (2018).

[39] L. Grünhaupt, M. Spiecker, D. Gusenkova, N. Maleeva, S. T. Skacel, I. Takmakov, F. Valenti, P. Winkel, H. Rotzinger, W. Wernsdorfer, A. V. Ustinov, and I. M. Pop, Granular aluminium as a superconducting material for high-impedance quantum circuits, Nat. Mater. 18, 816 (2019).

[40] A. J. Annunziata, D. F. Santavicca, L. Frunzio, G. Catelani, M. J. Rooks, A. Frydman, and D. E. Prober, Tunable superconducting nanoinductors, Nanotechnology 21, 445202 (2010).

[41] N. Samkharadze, A. Bruno, P. Scarlino, G. Zheng, D. P. DiVincenzo, L. DiCarlo, and L. M. K. Vandersypen, HighKinetic-Inductance Superconducting Nanowire Resonators for Circuit QED in a Magnetic Field, Phys. Rev. Appl. 5, 044004 (2016).

[42] T. M. Hazard, A. Gyenis, A. Di Paolo, A. T. Asfaw, S. A. Lyon, A. Blais, and A. A. Houck, Nanowire Superinductance Fluxonium Qubit, Phys. Rev. Lett. 122, 010504 (2019).

[43] G. Stan, S. B. Field, and J. M. Martinis, Critical Field for Complete Vortex Expulsion from Narrow Superconducting Strips, Phys. Rev. Lett. 92, 097003 (2004).

[44] O. V. Astafiev, L. B. Ioffe, S. Kafanov, Y. A. Pashkin, K. Y. Arutyunov, D. Shahar, O. Cohen, and J. S. Tsai, Coherent quantum phase slip, Nature 484, 355 (2012).
[45] P. Winkel, K. Borisov, L. Grünhaupt, D. Rieger, M. Spiecker, F. Valenti, A. V. Ustinov, W. Wernsdorfer, and I. M. Pop, Implementation of a Transmon Qubit Using Superconducting Granular Aluminum, Phys. Rev. X 10, 031032 (2020).

[46] W. C. Smith, A. Kou, U. Vool, I. M. Pop, L. Frunzio, R. J. Schoelkopf, and M. H. Devoret, Quantization of inductively shunted superconducting circuits, Phys. Rev. B 94, 144507 (2016).

[47] See Supplemental Material at http://link.aps.org/supple mental/10.1103/PhysRevApplied.14.064038, which contains a theoretical description of the uncoupled fluxonium and additional field data for devices $A$ and $B$.

[48] T. Yokoyama, M. Eto, and Y. V. Nazarov, Anomalous Josephson effect induced by spin-orbit interaction and Zeeman effect in semiconductor nanowires, Phys. Rev. B 89, 195407 (2014).

[49] D. B. Szombati, S. Nadj-Perge, D. Car, S. R. Plissard, E. P. A. M. Bakkers, and L. P. Kouwenhoven, Josephson $\phi_{0}$-junction in nanowire quantum dots, Nat. Phys. 12, 568 (2016).

[50] Y. Huang, H. Pan, C.-X. Liu, J. D. Sau, T. D. Stanescu, and S. Das Sarma, Metamorphosis of andreev bound states into majorana bound states in pristine nanowires, Phys. Rev. B 98, 144511 (2018).

[51] F. Dolcini, M. Houzet, and J. S. Meyer, Topological Josephson $\phi_{0}$ junctions, Phys. Rev. B 92, 035428 (2015).

[52] S. Wu, V. Fatemi, Q. D. Gibson, K. Watanabe, T. Taniguchi, R. J. Cava, and P. Jarillo-Herrero, Observation of the quantum spin hall effect up to 100 kelvin in a monolayer crystal, Science 359, 76 (2018).

[53] Y. Cao, D. Rodan-Legrain, O. Rubies-Bigorda, J. M. Park, K. Watanabe, T. Taniguchi, and P. Jarillo-Herrero, Tunable correlated states and spin-polarized phases in twisted bilayer-bilayer graphene, Nature 583, 215 (2020).

[54] X. Liu, Z. Hao, E. Khalaf, J. Y. Lee, Y. Ronen, H. Yoo, D. Haei Najafabadi, K. Watanabe, T. Taniguchi, A. Vishwanath, and P. Kim, Tunable spin-polarized correlated states in twisted double bilayer graphene, Nature 583, 221 (2020).

[55] C. W. J. Beenakker, Universal Limit of Critical-Current Fluctuations in Mesoscopic Josephson Junctions, Phys. Rev. Lett. 67, 3836 (1991).

[56] A. Bargerbos, W. Uilhoorn, C.-K. Yang, P. Krogstrup, L. P. Kouwenhoven, G. de Lange, B. van Heck, and A. Kou, Observation of Vanishing Charge Dispersion of a Nearly Open Superconducting Island, Phys. Rev. Lett. 124, 246802 (2020).

[57] A. Kringhøj, B. van Heck, T. W. Larsen, O. Erlandsson, D. Sabonis, P. Krogstrup, L. Casparis, K. D. Petersson, and C. M. Marcus, Suppressed Charge Dispersion via Resonant Tunneling in a Single-Channel Transmon, Phys. Rev. Lett. 124, 246803 (2020). 